\title{
Development of broadband infrared single-mode fibers for the DARWIN mission
}

\author{
Lun K. Cheng ${ }^{* a}$, Anne-Jans Faber ${ }^{\mathrm{a}}$, Wim Gielesen ${ }^{\mathrm{a}}$, Jacques Lucas ${ }^{\mathrm{b}}$, Catherine Boussard-Plédel ${ }^{\mathrm{b}}$, \\ Patrick Houizot ${ }^{\mathrm{b}}$, João Pereira Do Carmo ${ }^{\mathrm{c}}$ \\ ${ }^{a}$ TNO Science and Industry, P.O. Box 155, 2600 AD Delft, The Netherlands; \\ ${ }^{b}$ University of Rennes, Glass and Ceramic Laboratory, Campus of Beaulieu, 35042 Rennes, Frans; \\ ${ }^{c}$ European Space Agency, P.O. Box 299, 2200 AG Noordwijk ZH, The Netherlands
}

\begin{abstract}
A vital function of the space interferometer foreseen in the DARWIN mission is the so-called "nulling" operation. The challenge of nulling is making the null in the interferometric signal sufficiently deep to cancel the light from the bright star during the collection of light from its surrounding planets. The performance of the nulling is limited by the wavefront quality of the beams. The wavefront error can be reduced by filtering. One promising concept for nulling wavefront filtering is using a single mode fiber. For the wavefront filtering in the DARWIN mission, the fiber has to cover the operational wavelength range of 4-20 $\mu \mathrm{m}$. Furthermore, a minimal insertion loss is required to ensure a minimum exposure time. This results in the separation of the complete wavelength range into several separate wavelength bands in the nulling system. Within an ESA project, a chalcogenide glass fiber based on the Te-As-Se (TAS) composition is selected to be used for the short wavelength band. TNO has designed and tested several TAS fibers that have been manufactured by the University of Rennes. Single mode operation is demonstrated. Furthermore, the effect of bending the fiber and light coupling are investigated. For the long wavelength band up to $20 \mu \mathrm{m}$, Tellurium based glass is proposed. Different samples of various composition based on Te glass are manufactured. Accurate temperature control to avoid crystallization is found to be essential for the manufacturing process. For the bulk material, a transmission window up to $20 \mu \mathrm{m}$ is measured.
\end{abstract}

Keywords: fiber optic, interferometer, interferometry, astrometry

\section{INTRODUCTION}

Various space telescope array systems are being considered to investigate other terrestrial planets orbiting around nearby stars in order to find extra-terrestrial life. One of them is the DARWIN mission of the European Space Agency (ESA). The star/planet systems of interest are those with stars having properties similar to our Sun and with planets located at a reasonable distance from the star in a way similar to our Earth in the so-called habitable zone. System issues such as the available flux from the stars and the possible size of the satellites restrain the search zone to nearby stars located at a distance around 20 parsec. Seen at this distance the angular separation between our Sun and Earth is 50 milli-arcsec. A telescope wanting to separate these two objects at a wavelength of 5 microns would need a diameter of $50 \mathrm{~m}$. A smaller diameter could be sufficient at visible wavelengths. However in the visible spectrum the signal from the planet is much smaller with respect to the star signal than in the thermal infra-red. Because in the visible the planet only reflects a part of the light emitted by the star whereas in the thermal infra-red it has a proper thermal emission. This imposes the choice of the long wavelengths and thus the need for large telescopes. A monolithic telescope with a $50 \mathrm{~m}$ diameter is presently not considered as a feasible option. This led to the proposal to use aperture synthesis technology. Small telescopes separated by a $50 \mathrm{~m}$ distance will have the same resolving power providing the light of the telescopes are recombined coherently.

As the flux emitted by a star is extremely large compared to the flux emitted by orbiting planets, a so-called "nulling" operation is vital for DARWIN. Without nulling, the star signal would completely mask the planet signal. Nulling is based on the principle of destructive interference of the star signal. The planet signal is not nulled because the planet is slightly off axis so that the phase between the various beams is different from that of the star. This trick reduces the 
contrast between star and planet and enables the detection of the planet. The challenge of nulling is making the null sufficiently deep (nulling ratio $>10^{6}$ ).

Nearly perfect wavefront of the beams is required to achieve sufficient nulling. This can be realized by wavefront error filtering before the detection. Single mode fiber is one promising candidate for the wavefront filtering [1]. For the application of the single mode fiber as wavefront filter in DARWIN, a minimal insertion loss is desired (total insertion loss $<1.5 \mathrm{~dB})$. The single mode fiber to be developed for DARWIN shall operate in the infrared spectral region $(4-20$ $\mu \mathrm{m})$. Furthermore, the fiber has to meet the operational condition in space like sustain the low operational temperature $(40 \mathrm{~K})$ and not to be radiation sensitive.

In Section 2, the suitable materials are discussed and optical properties of bulk glass samples of different compositions are presented. The optical measuring data is used to design core-cladding fiber structures with desired single mode wave propagation properties (Section 3). In Section 4 the test results of the fiber is presented. The development of new highTe glass material is shown in Section 5.

\section{MATERIALS FOR THE INFRARED SINGLE MODE FIBER}

Since the main emission lines of the relevant atmospherical components like $\mathrm{CO}_{2}, \mathrm{O}_{3}$ and water vapor are all in the mid IR, from $4-20 \mu \mathrm{m}$, the envisaged single mode fiber must be transparent in this spectral range. The candidate infrared transmitting glasses can be divided in:

- Halide glasses based on chlorides, bromides and iodides (like $\mathrm{ZnCl}_{2}, \mathrm{ZnBr}_{2}, \mathrm{BiCl}_{3}, \mathrm{KCl}, \mathrm{KBr}$ and $\mathrm{CdCl}_{2}$ ) [2]

- Chalcogenide glasses, i.e. glassy materials containing one or more of the chalcogen elements $\mathrm{S}, \mathrm{Se}$ or $\mathrm{Te}$ (sulphide, selenide and telluride glasses). [3]

Next to specifications with respect to IR transmission, the candidate glasses must satisfy technological requirements with regard to the viscosity behavior and chemical stability, enabling the manufacturing of single mode, core-cladding fibers.

In general, chalcogenide glasses have a better chemical stability, better mechanical properties and better fiber drawing potential than halide glass types. Therefore, chalcogenide glasses were selected for the development and demonstration of an IR single mode fiber operating as a wavefront filter in the short wavelength range of the DARWIN spectral range.

For the chalcogenide glasses, Te-based glasses have basically the highest transmission in the IR. However, these glasses tend to be less stable towards crystallisation. Selenide glasses, especially Te-As-Se-glasses have a broad IR transparency range, acceptable thermo-mechanical properties and good chemical stability. Therefore, the development of the IR single mode fiber is started with the TAS glasses.

\subsection{Optical properties}

For the development of IR single mode fiber, both the spectral absorption/transmission and the refractive index of the material are of vital importance. The results of the refractive index measurement of the TAS material were presented earlier [1]. The spectral absorption was determined from the ratio of two transmission spectra, $T_{1}$ and $T_{2}$, recorded for samples of different thickness, $d_{1}$ and $d_{2}$, using as first estimate for the spectral extinction coefficient $K(\lambda)$ :

$$
\mathrm{K}_{1}(\lambda)=\frac{1}{\mathrm{~d}_{2}-\mathrm{d}_{1}} \ln \left|\frac{\mathrm{T}_{1}(\lambda)}{\mathrm{T}_{2}(\lambda)}\right|
$$

Taking into account multiple reflections in the sample, the following formula was used, representing subsequent iteration steps for the extinction coefficient: 


$$
\mathrm{K}_{\mathrm{i}}(\lambda)=\frac{1}{\mathrm{~d}_{2}-\mathrm{d}_{1}}\left[\ln \left|\frac{\mathrm{T}_{1}(\lambda)}{\mathrm{T}_{2}(\lambda)}\right|+\ln \left|\frac{1-\mathrm{R}^{2}(\lambda) \exp \left\{-2 \mathrm{~K}_{\mathrm{i}-1}(\lambda) \mathrm{d}_{1}\right\}}{1-\mathrm{R}^{2}(\lambda) \exp \left\{-2 \mathrm{~K}_{\mathrm{i}-1}(\lambda) \mathrm{d}_{2}\right\}}\right|\right]
$$

where $\mathrm{R}(1)$ is the single surface reflection.

The iteration procedure converges and is stopped at a final value of $\mathrm{K}(\lambda)$ that is obtained when the difference between two consecutive values for $\mathrm{K}_{\mathrm{i}}(\lambda)$ is negligible $\left(<10^{-4} \mathrm{~K}_{\mathrm{i}}\right)$. The advantage of this transmittance ratio method (as compared to using a single transmittance) is that systematic errors are reduced significantly [4].

The results of transmission measurements of a highly purified TAS material are shown in the figure below. Bulk material of two different thickness' $(1 \mathrm{~mm}$ and $3 \mathrm{~mm})$ are measured before and after radiation of $0.1 \mathrm{kGy}$. The influence of thickness is obvious while no radiation susceptibility is found for this highly purified TAS glass.

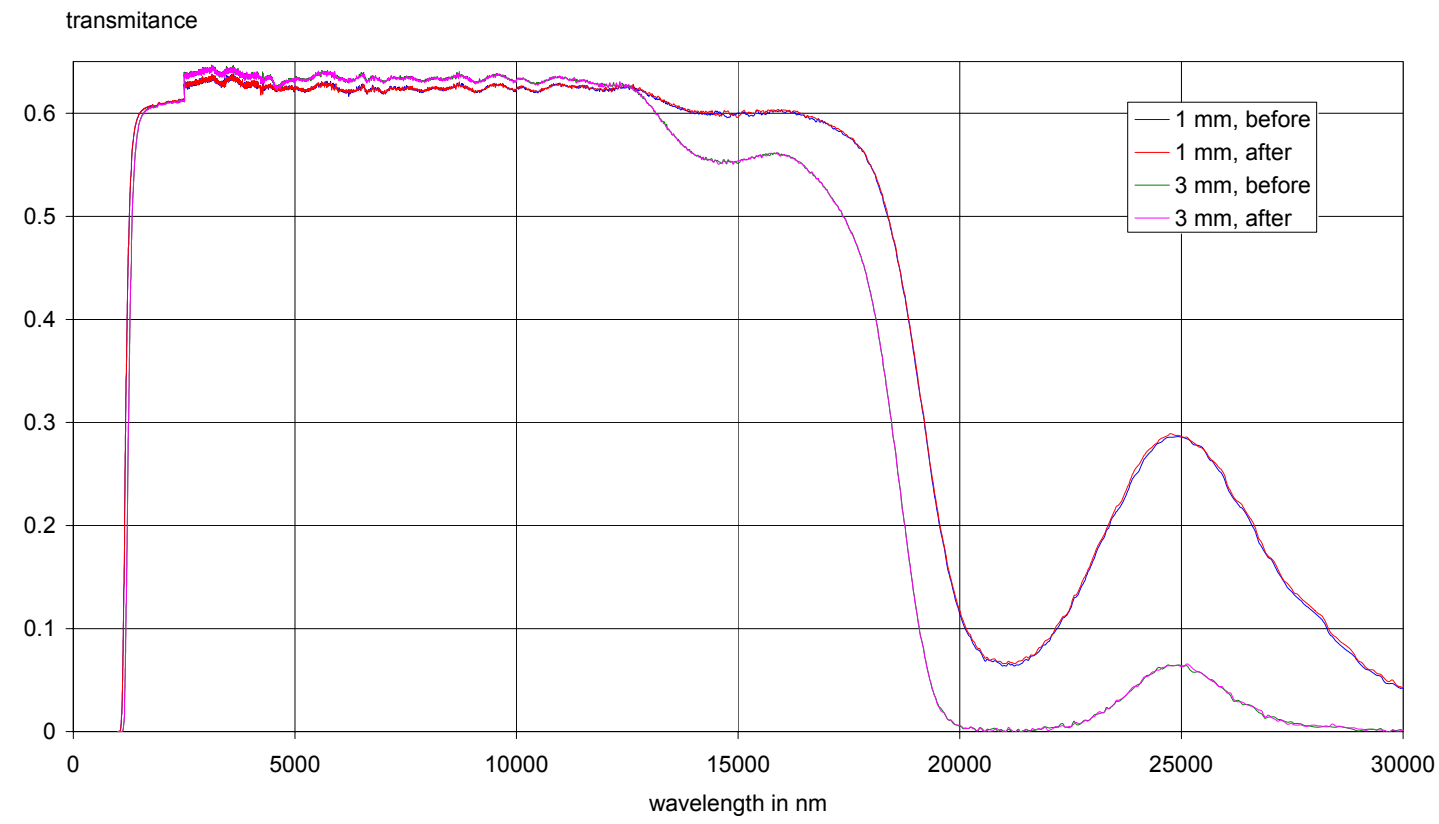

Fig. 1. Spectral curves of the transmittance of the TAS samples of 1 resp. $3 \mathrm{~mm}$ thickness, before and after radiation treatment.

The calculated spectral absorption of the highly purified TAS glass is presented in Fig. 2. To demonstrate the importance of the purification, the spectral absorption of the same TAS glass manufactured using standard purification process is also shown. TAS material manufactured using standard purification process has two extra absorption bands around 13 and $15 \mu \mathrm{m}$ (probably related to vibration of the As-O-As bridges) which can be removed by extra purification. Only the relatively weak broad multi-phonon vibration band As-Se glass matrix around $14 \mu \mathrm{m}$ remains. The usefulness of this TAS material between 13 and $17 \mu \mathrm{m}$ depends on the required length of the single mode fiber for wavefront filtering. For the current TAS glasses, the absorption increases significantly for wavelength longer than 17 $\mu \mathrm{m}$. Other material has to be found to cover the wavelength band of $17-20 \mu \mathrm{m}$. First test results with high Te-glasses are presented in Section 5 . 


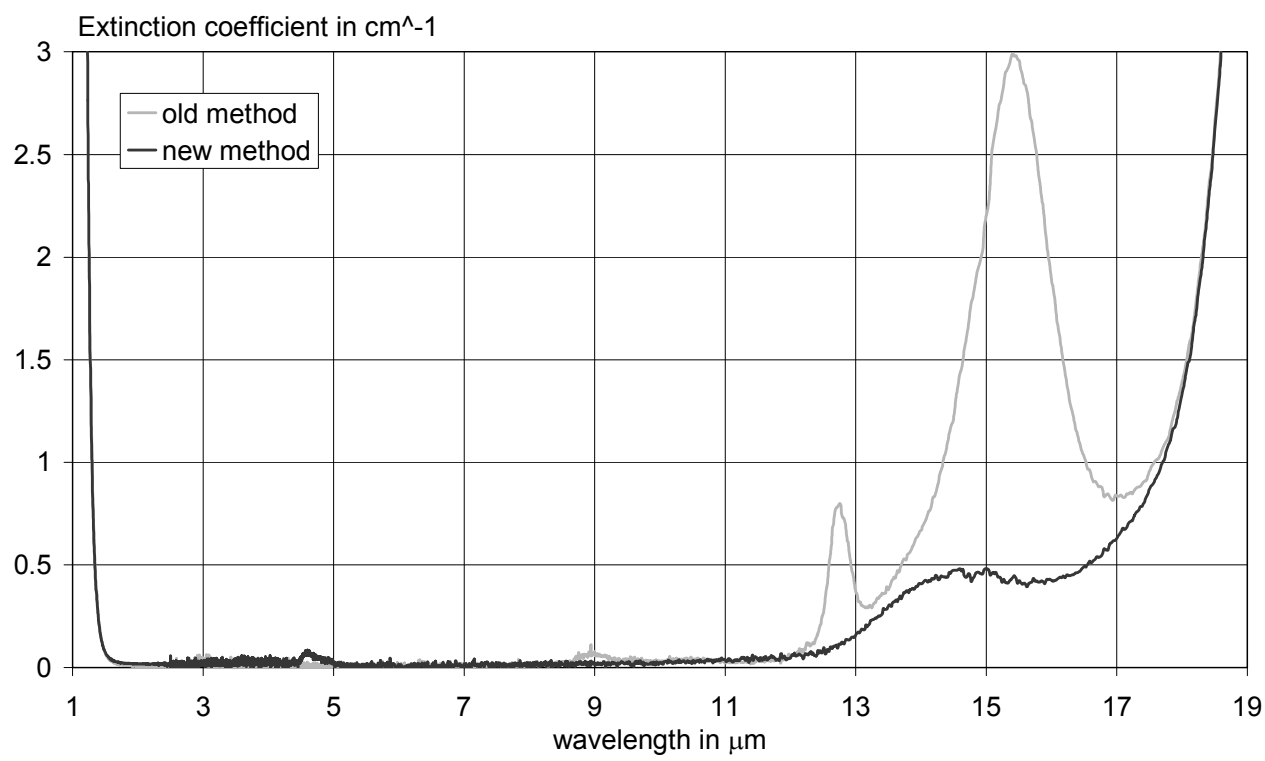

Fig. 2. Spectral absorption of TAS glass. Old method: using standard purification process. New method: using highly purification process.

\section{DESIGN AND MANUFACTURING OF THE TAS FIBERS}

\subsection{Design of the TAS single mode fiber}

Theoretically, a conventional step index fiber can be used for a wide wavelength range. A practical requirement is that the mode diameter has to be much smaller than the cladding diameter of the fiber to ensure the confinement of the mode. However, the longer the wavelength, the more the mode field stretches into the cladding and the more sensitive to bending the fiber becomes. For the development of a baseline design of the single mode fiber for Darwin, the following issues have to be investigated:

- Insertion loss, in particular the coupling efficiency as function of the wavelength

- Minimum length for sufficient higher-order mode suppression

The problem of insertion loss was evaluated on the basis of the relation between the mode field diameter (MFD) and the wavelength. The MFD is defined as the value where the mode field is dropped to $1 / \mathrm{e}^{2}$ of the maximum value. The ideal broadband single mode fiber for the DARWIN mission should have a MFD with is proportional to the wavelength because the diameter of the focusing spot is linear with the wavelength. However, the MFD of a step-index single mode fiber increases drastically for longer wavelength. Consequently, the coupling efficiency drops significantly for longer wavelengths. Therefore, a dual band system consisting of two different single mode fibers for each wavelength band has been proposed: the short wavelength (SW) and long wavelength (LW) fiber. Using the refractive index and dispersion data of the TAS material achieved on bulk sample testing, a final design fine tuning resulted in the following main parameters for a dual band configuration:

Table 1 Dual wavelength band system.

\begin{tabular}{|l|c|c|}
\hline & Short wavelength $(\mathrm{SW})$ & Long wavelength $(\mathrm{LW})$ \\
\hline Wavelength range $(\mu \mathrm{m})$ & $4-9$ & $>9$ \\
\hline Cut off $(\mu \mathrm{m})$ & 3.7 & 8.5 \\
\hline Core radius $(\mu \mathrm{m})$ & 11 & 26 \\
\hline Cladding thickness $(\mu \mathrm{m})$ & 250 & 600 \\
\hline n core & 2.927 & 2.913 \\
\hline n cladding & 2.924 & 2.910 \\
\hline
\end{tabular}


For the short wavelength (SW) TAS fiber, the relation between the MFD and the wavelength is modeled and presented in Fig. 3.

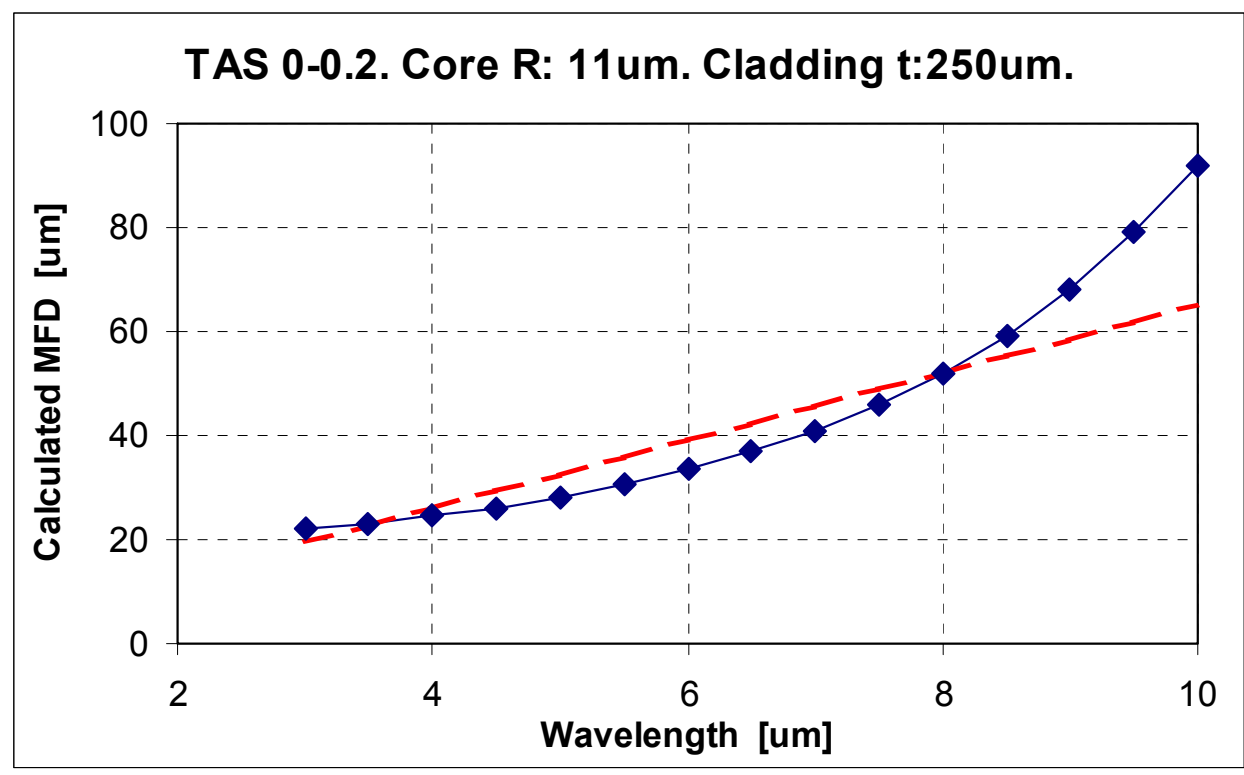

Fig. 3. Calculated MFD of the Short wavelength fiber as a function of the wavelength (blue line with markers). The red dashed line presents the desired linear relation between the MFD and the wavelength for optimized coupling @ $8 \mu \mathrm{m}$.

The coupling efficiency as function of the wavelength for coupling conditions optimized for different wavelengths is investigated and the results are shown in Fig. 4. For the configuration in which the coupling efficiency is optimized for $8 \mu \mathrm{m}$, the coupling efficiency over the operational wavelength range of $4-9 \mu \mathrm{m}$ is found to be higher than $80 \%$.

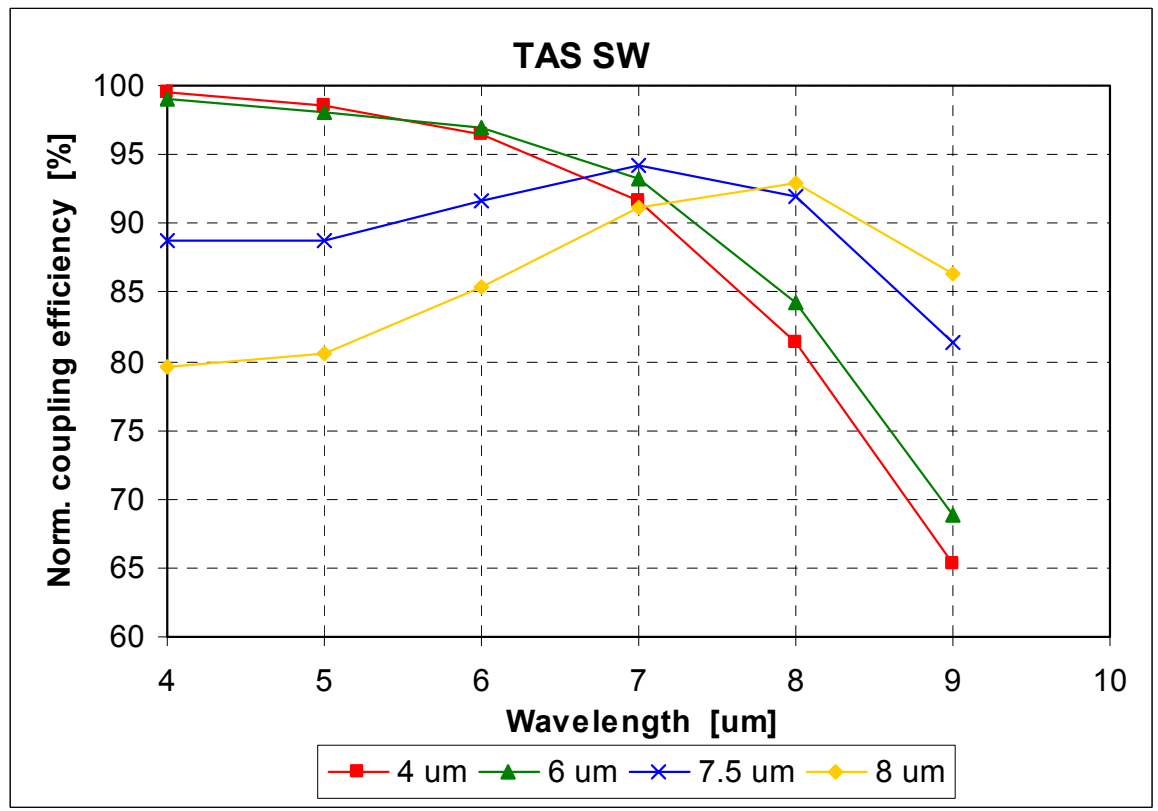

Fig. 4. Coupling efficiency of the proposed SW TAS 0-0.2 fiber as a function of the wavelength. 


\subsection{Manufacturing methods for the IR single mode fibers}

Different manufacturing techniques are developed and used by the University of Rennes for the fabrication of the TAS fibers.

\subsubsection{Internal rotational casting method (IRCM)}

The first TAS fiber (TAS-1) is manufactured by the Internal rotational casting method (IRCM) which is patented by the University of Rennes. In this method the core/clad preform is made with a silica set-up as shown schematically in Fig. 4. The silica ampoule is cleaned with hydrofluoric acid solution, rinsed with distilled water and dried under vacuum. The core and the cladding glasses are introduced in the appropriate amounts. The silica ampoule is placed in a furnace in a special position to avoid the mixing of the core and cladding glasses and heated at $500^{\circ} \mathrm{C}$ to melt the glasses. Then the silica ampoule is rotated counter clockwise at room temperature to pour the tube in vertical position and immediately the tube part is quenched in water during a few seconds. The time of the quenching defines the internal diameter of the cladding. A clockwise rotation permits to empty the center of the rod that is not yet solidified. To finish, another clockwise rotation is applied to fill the clad tube by the core glass and the complete silica system is annealed around the glass transition temperature. Test results of the TAS fiber are shown in Section 4.

\section{1 : Pouring with the clad glass \\ 2: Quench in water}

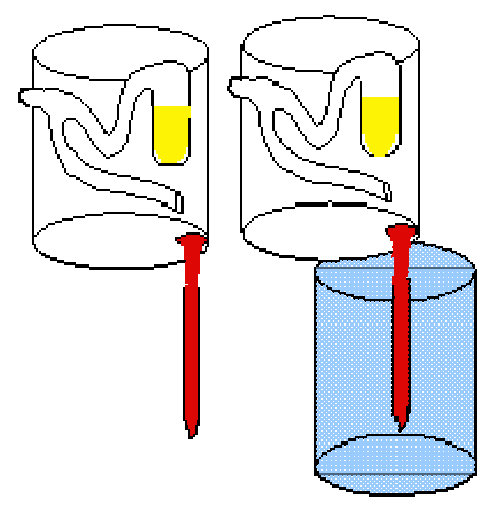

\section{3 : Rotation at $90^{\circ}$ to empty the glass not yet solidified}

\section{$4: 2^{\text {nd }}$ rotation at $90^{\circ}$ \\ to fill the clad tube \\ with core glass}
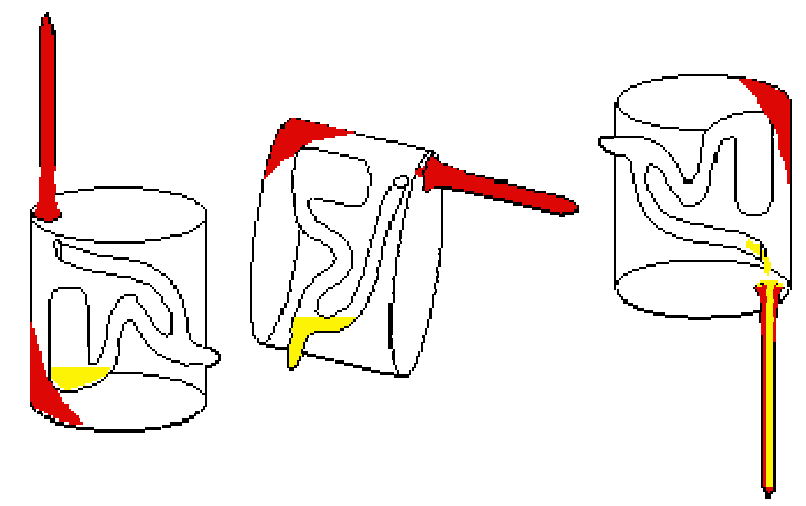

Fig. 5. Internal rotation casting method (IRCM).

Because the reproduction of the IRCM technique proved to be rather complex, time consuming and operator dependant, a second technique was explored, designated as Fiber in Tube Vacuum Method (FTVM) to manufacture the next short wavelength range TAS fiber.

\subsubsection{One step fiber in tube vacuum method (FTVM)}

For the one-step fiber in tube method, a core glass fiber with a diameter of $400 \mu \mathrm{m}$ is inserted in a cladding glass tube with an external diameter of $10 \mathrm{~mm}$ and an internal diameter between $2.8 \mathrm{~mm}$ and $4 \mathrm{~mm}$. A schematic drawing of this method is shown in Fig. 6.

The first fiber samples achieved experimentally (TAS-X), applying the FTVM technique for the first time on TAS material, showed a Gaussian, circularly shaped far field intensity distribution at $10.6 \mu \mathrm{m}\left(\mathrm{CO}_{2}\right.$-laser $)$. Microscopic inspection of cross section of a fiber manufactured by FTVM showed that the interface between core and cladding glass is not visible, indicating a good bonding, and that the material is free of air bubbles, inclusions and irregularities. Disadvantage of the FTVM method is the poor reproducibility of the geometry (diameter, circularity) of the core due to 
the large difference in diameter between core-fiber and clad-tube. To improve the control of fiber geometry for this new method, the strong elements of both the IRCM and the FTVM method are combined, resulting in the development of the manufacturing of TAS fibers into the Rod in Tube Vacuum Method (RTVM).

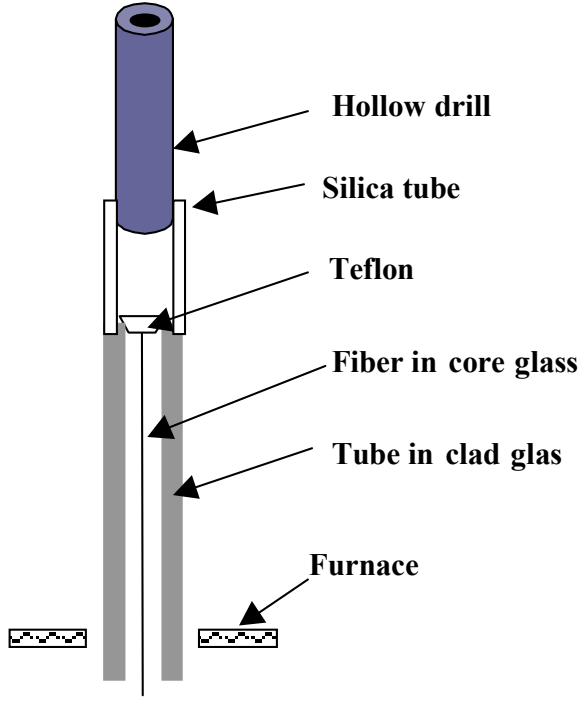

(a)

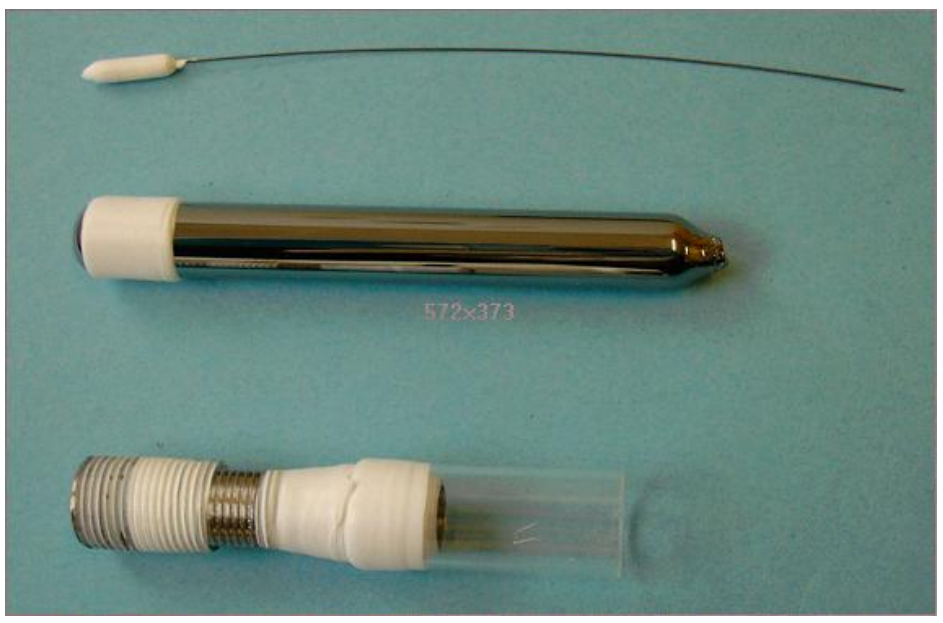

(b)

Fig. 6. (a) One step fiber in tube vacuum method (FTVM). (b) Different parts of the preform for FTVM; core glass fiber (on top), cladding glass tube (middle) and glass tube for fixation of cladding glass tube to the fiber drawing set-up

\subsubsection{Rod in Tube Vacuum Method (RTVM)}

In the RTVM method, the fiber with a diameter of $400 \mu \mathrm{m}$ for the forming of the core is replaced by a rod with a diameter of $2 \mathrm{~mm}$. The smaller difference between the rod diameter and the internal diameter of the tube prevents the deformation of the core during the collapsing of the tube during the fiber drawing process. The RTVM technique is a 3step process:

1. Preparation of TAS core glass rod and cladding glass tube

2. Preparation of intermediate core-cladding rod

3. Final preform preparation and fiber drawing

At first, a high contrast fiber (HC-TAS-2) was drawn to demonstrate the quality of the core geometry achieved by applying this RTVM technique. The cross section of this fiber showed a good circular shape of the core. With this result, finally fiber TAS\#3 was manufactured with RTVM.

\section{TEST RESULTS OF THE IR SINGLE MODE FIBERS}

The following tests have been carried out to characterize the quality and performance of TAS fibers:

- Visual inspection

- Determination of the cut-off wavelength (Mode Field Diameter (MFD) measurement)

- Determination of the presence of higher order modes (Intermodal Interference Measurement (IIM))

- Far-field intensity measurement

- Attenuation measurement 


\subsection{Far-field intensity measurement at $10.6 \mu \mathrm{m}$}

A set-up was developed at TNO to measure 2D far-field intensity distribution at $10.6 \mu \mathrm{m}$ using a $\mathrm{CO}_{2}$ laser and a $2 \mathrm{D}$ IR camera. With this set-up, a number of tests on different TAS fibers were performed to show the influence of the following parameters on the performance of a TAS-fiber:

- Presence and quality of absorbing coating for stripping of cladding modes

- Length of fiber necessary to suppress higher order modes in order to achieve single mode operation

- Effect of bending the fiber

- Mismatching of light coupling

The first two items are discussed in a previous paper [1]. Single mode operation of different TAS fibers with absorbing coating is presented (See Fig. 7).
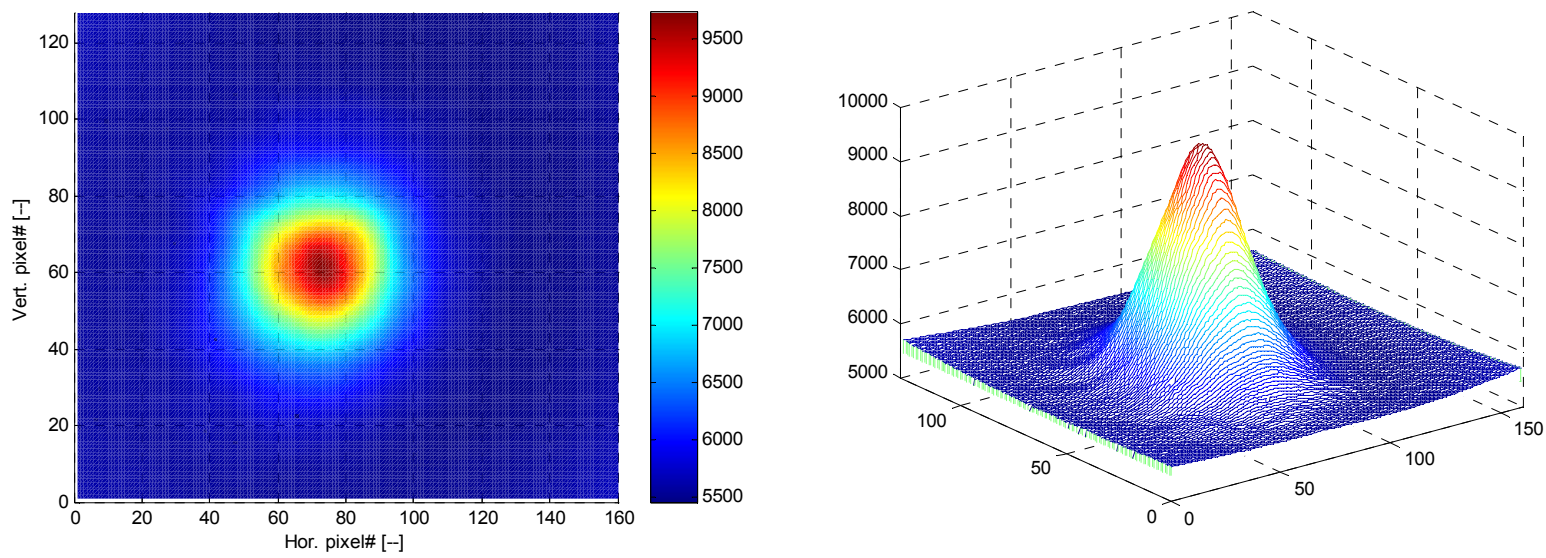

Fig. 7. Far-field intensity distribution of TAS-X fiber demonstrates single-mode operation and a circular intensity distribution.

However, the intensity distribution of the first TAS-1 fiber is found to be elliptical. This is probably caused by nonrotational symmetric mechanical stress in the fiber introduced by winding the fiber on a drum (about $50 \mathrm{~cm}$ diameter) directly after the drawing process. The influence of the mechanical stress is demonstrated by bending the fiber (See Fig. 8).

\subsection{Effect of bending the fiber}

Some tests to demonstrate the influence of bending the TAS fiber on the far-field intensity distribution are performed. Bending the fiber will introduce mechanical stress in the fiber, which is one of the possible reasons for the elliptical farfield intensity distribution of the TAS-1 fiber. The TAS-1 fiber has a curvature due to the production process. The radius is about $30 \mathrm{~cm}$. A section of $65 \mathrm{~mm}$ of a TAS-1 fiber is bent to straight while the far-field intensity distribution is measured. The results are shown in Fig. 7 and the influence of bending on the far-field intensity distribution can clearly be observed. For the application in the DARWIN mission, mechanical disturbance on the fiber has to be avoid to ensure a stable output beam from the IR fiber. 


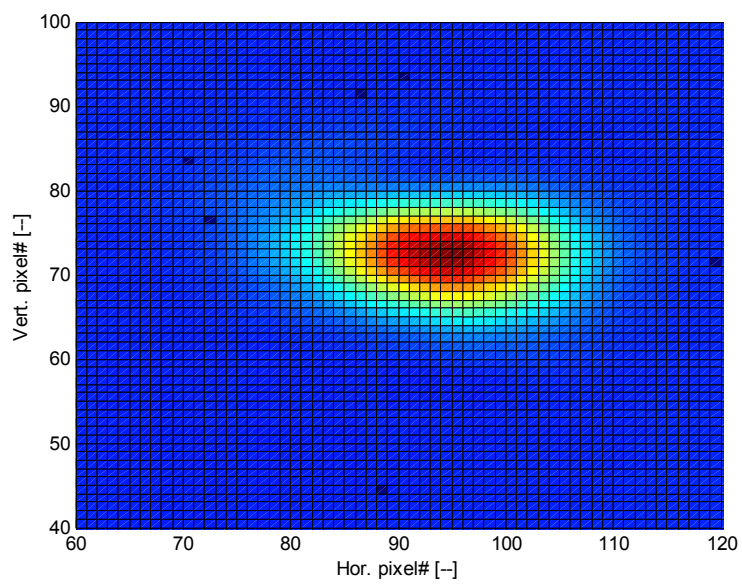

(a)

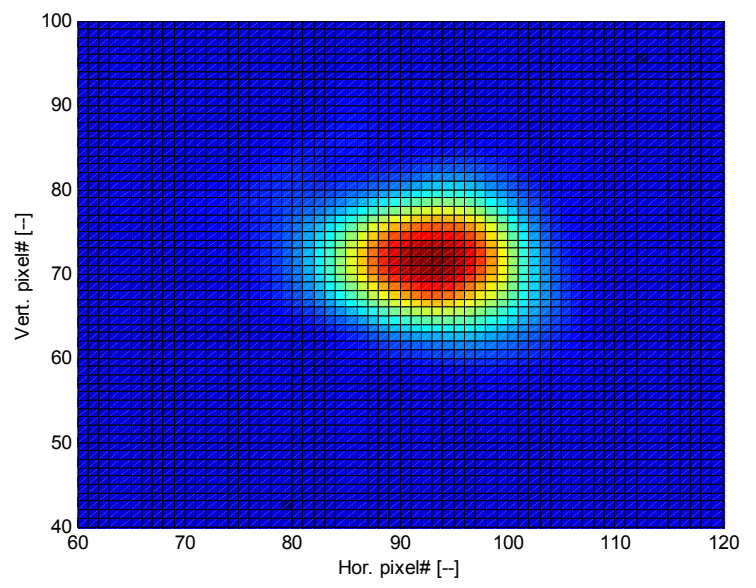

(b)

Fig. 8. Effect of bending the TAS fiber TAS1-F on the far-field intensity distribution. (a) No bending. (b) Section of $65 \mathrm{~mm}$ is bent.

\subsection{Mismatching of light coupling}

A test of mismatching of light coupling in a single mode TAS-1 is carried out by translating the fiber in respect to the pinhole in 3 steps of $20 \mu \mathrm{m}$ in the horizontal direction $\mathrm{x}$. Light will be gradually coupled into the cladding. Due to the Gallium coating, the cladding modes are stripped almost completely for $x=60 \mu \mathrm{m}$. The amplitude of the peak in the different situations are measured and presented in Fig. 9. The effect of mismatching on the amount of energy coupled into the fundamental mode of the single mode fiber is demonstrated. Since the performance of the nulling also depends on the amplitude of the beams in the nulling interferometer, a high mechanical stability of the fiber positioning mechanism will be required.

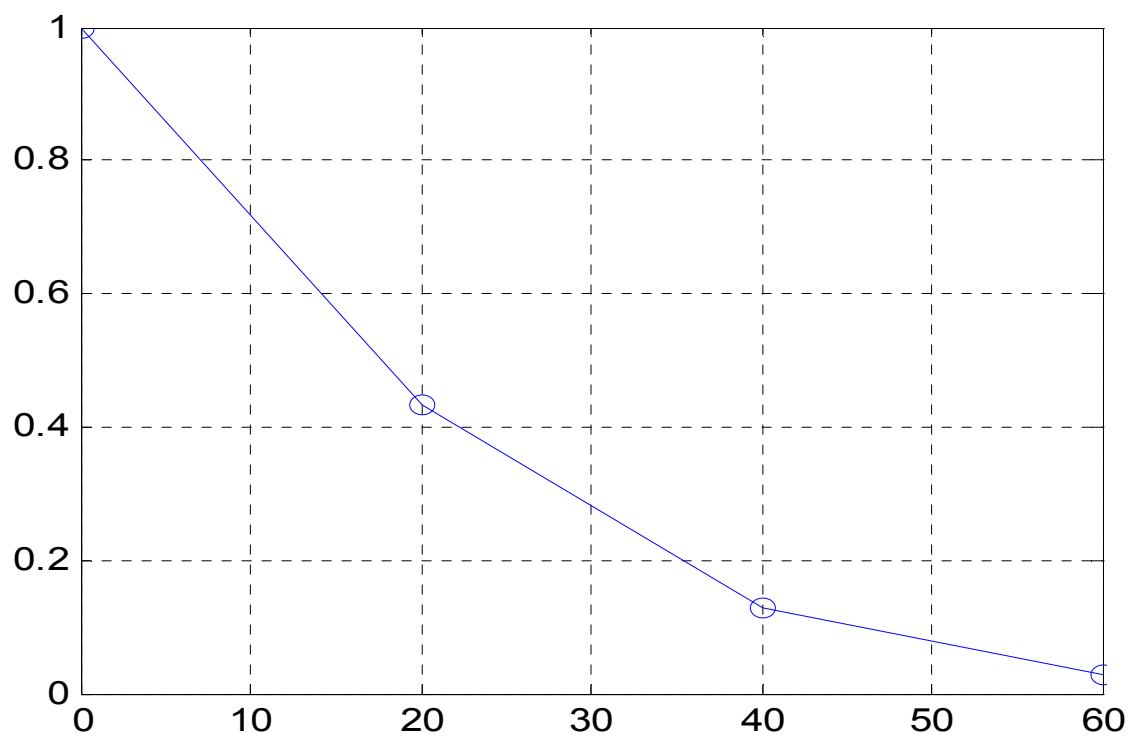

Fig. 9. Relative peak amplitude as function of mismatching of light coupling. 


\section{MATERIAL FOR LONG WAVELENGTH RANGE UP TO $20 \mu \mathrm{m}$}

Due to the absorption bands around $14 \mu \mathrm{m}$ and $20 \mu \mathrm{m}$ caused by As-Se vibrational modes in the glass matrix, the useful wavelength range of the TAS fiber cannot reach $20 \mu \mathrm{m}$. For the long wavelength band up to $20 \mu \mathrm{m}$, other material has to be used. Therefore, a study was started to develop a new high Te glass fiber. Different samples of various compositions based on high Te glass are manufactured. For this type of glasses, the range $\Delta \mathrm{T}$ between the glass temperature $\mathrm{Tg}$ at which the viscosity increases drastically and the temperature Tx at which crystallisation starts to occur is limited. Accurate temperature control to avoid crystallization is therefore essential for the manufacturing process. $\Delta \mathrm{T}$ is an excellent indicator of the glass stability. This parameter is very important for the fiber drawing process and is used for the selection of the suitable glass. A large number of materials of different composition using Germanium, Gallium and Iodine are manufactured for the selection of the composition with a sufficient $\Delta \mathrm{T}$.

\subsection{Results of Te\#01 glass}

The spectral transmission of one of the high Te glass Te\#01 is shown in the figure below. For a sample without special purification, a significant absorption band around $14 \mu \mathrm{m}$ is visible. This peak is caused by the Ge-O vibrational bond, due to the presence of oxygen impurities and hence considered as extrinsic to the material. In order to check the validity of this hypothesis, a sample with special purification is manufactured and measured. It can be clearly seen that the oxygen metallic vibrational bond around $14 \mu \mathrm{m}$ has totally disappeared. The importance of a dedicated purification process is demonstrated again. The absorption shoulder around $22 \mu \mathrm{m}$ is probably caused by multi-phonon absorption and is considered as intrinsic to the material.

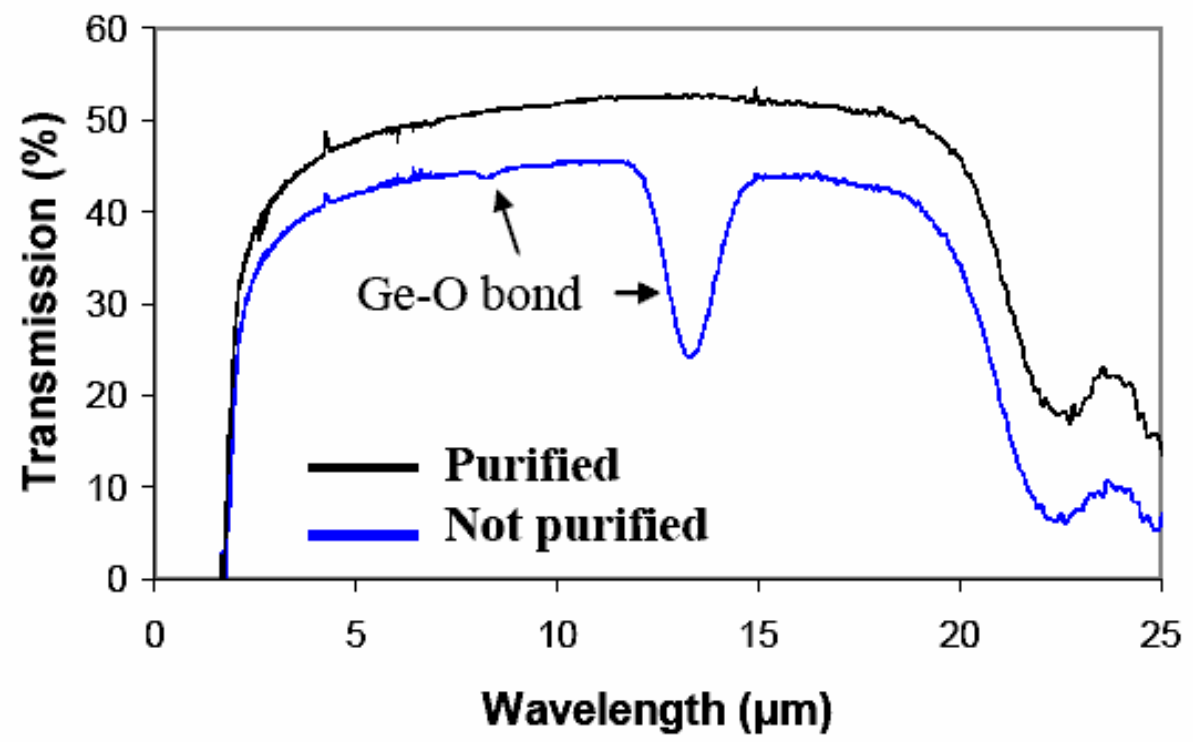

Fig. 10. Transmission spectra of purified and non-purified Te\#01 high-Te glass.

In comparison to the transmission spectrum of the highly purified TAS glasses (Fig. 1), the purified Te\#01 glass doesn't have the broad multi-phonon vibration band from 13 to $17 \mu \mathrm{m}$ and the transmission window of this material is found to be up to $20 \mu \mathrm{m}$.

$\Delta \mathrm{T}$ of the Te\#01 material is measured to be $>100 \mathrm{deg}$. $\mathrm{C}$ and is potentially suitable for fiber drawing. However, great care of temperature control has still to be taken to avoid crystallisation. Mono-index fibers based on both the purified and non-purified Te\#01 material are drawn and spectral attenuation of the fibers are measured using the cut-back method. The effect of purification on the attenuation can also be observed clearly. 


\section{CONCLUSIONS}

Results on the development of IR single mode fibers to operate as wavefront filtering in the DARWIN mission are presented. Chalcogenide glass based on Te-As-Se composition is selected to be a promising material to cover a large part of the spectrum between 4-20 $\mu \mathrm{m}$. Different compositions are investigated and the importance of purification is demonstrated. The presented TAS glass has high transmission up to $13 \mu \mathrm{m}$. A weak broad multi-phonon vibration band As-Se glass matrix occurs around $14 \mu \mathrm{m}$. The usefulness of this TAS material between 13 and $17 \mu \mathrm{m}$ depends on the required length of the single mode fiber for wavefront filtering. For the current TAS glasses, the absorption increases significantly for wavelength longer than $17 \mu \mathrm{m}$. High Te-glass is supposed to have high transmission for wavelength even longer than $17 \mu \mathrm{m}$. The purified Te\#01 glass shows a large transmission window from about $4 \mu \mathrm{m}$ to about $20 \mu \mathrm{m}$. $\Delta \mathrm{T}$ of the Te\#01 material is measured to be $>100 \mathrm{deg}$. $\mathrm{C}$ and is potentially suitable for fiber drawing. However, great care of temperature control has to be taken to avoid crystallisation.

Different manufacturing techniques developed and used by the University of Rennes for the fabrication of the TAS fibers are discussed. Using far-field intensity distribution measurement, single mode operation of Gallium coated TAS fibers are demonstrated. The effect of fiber bending of the far-field intensity distribution reveals the influence of mechanical stress. Furthermore, the effect of mismatching on light coupling on the output intensity is shown.

\section{ACKNOWLEDGEMENT}

The funding for the development of the IR single mode fiber based on TAS and high Te-glass for wavefront filtering by the European Space Agency is acknowledged.

\section{REFERENCES}

1. L.K. Cheng, A.J. Faber, W. Gielesen, C. Boussard-Pledel, P. Houizot, J. Lucas and J.P. Do Carmo, "Test results of the infrared single-mode fiber for the DARWIN mission", Techniques and Instrumentation for Detection of Exoplanets II, Editor D.R. Coulter, Vol. 5905, 2005.

2. J.Lucas, "Halide glasses", Materials Sciences and Technology, Editor Zarsiky, Vol.9, pages 457-488, 1991.

3. S.R. Elliot, "Chalcogenide Glasses", Materials Sciences and Technology, Editor Zarsiky, Vol.9, pages 377-448, 1991.

4. P.A. van Nijnatten, "Accurate measurement of absorption spectra and refractive index of glass by spectrophotometry”, Glastech.Ber. Glass Sci. Technol. 77 C, pages 136-148, 2004. 